\title{
Short-term dynamics of microplankton abundance and diversity in NW Mediterranean Sea during late summer conditions (DYNAPROC 2 cruise; 2004)
}

\author{
S. Lasternas ${ }^{1,2, *}$, A. Tunin-Ley ${ }^{1,2}$, F. Ibañez ${ }^{1,2}$, V. Andersen ${ }^{1,2}$, M.-D. Pizay ${ }^{1,2}$, and R. Lemée ${ }^{1,2}$ \\ ${ }^{1}$ Université Pierre et Marie Curie-Paris6, Laboratoire d'Océanographie de Villefranche, 06230 Villefranche-sur-Mer, France \\ ${ }^{2}$ CNRS, Marine Microbial Ecology and Biogeochemical Group, Laboratoire d'Océanographie de Villefranche UMR 7093, \\ 06230 Villefranche-sur-Mer, France \\ * present address: IMEDEA (CSIC-UIB), Instituto Mediterráneo de Estudios Avanzados, Department of Global Change \\ Research, 07190 Esporles (Mallorca), Spain
}

Received: 23 September 2008 - Published in Biogeosciences Discuss.: 22 December 2008

Revised: 31 January 2011 - Accepted: 19 February 2011 - Published: 22 March 2011

\begin{abstract}
Changes in marine phytoplankton communities at short-time scales have rarely been examined. As a part of the DYNAPROC 2 cruise (14 September-17 October 2004), conducted in the NW Mediterranean Sea, we daily sampled and determined taxonomic composition and abundance of the microphytoplankton and the dominant microzooplanktonic groups from both net and bottle sampling, during 4 cycles of 5 days. Hydrological conditions were characterised by the dominance of a stratified water column and nutrientdepleted conditions. However the stratification index revealed a destabilization of the water column from the beginning of the second cycle, related to a wind stress event accompanied with coastal water intrusion. This conducted to an increase of taxonomic richness and a general decrease of evenness, depicting the dominance of species with the best fitness. We also emphasised on the great interest of taxonomic studies, as able to provide valuable information on biogeochemical-important groups of species, potential water masses indicators and trophic aspects of the community that are ignored or largely underestimated with other kind of phytoplankton studies, such as pigment analysis.
\end{abstract}

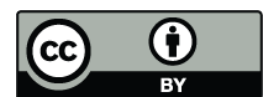

Correspondence to: R. Lemée (lemee@obs-vlfr.fr)

\section{Introduction}

Marine phytoplankton constitutes the base of the pelagic food-web and is responsible for about half of the net annual primary production on Earth (Field et al., 1998; Falkowski et al., 2004). Current studies on monitoring marine phytoplankton are mainly based on satellite-derived values from remote sensing (Kyewalyanga et al., 2007) or deal with the pigment diversity (Marty et al., 2002), rather than examining changes in diversity or species composition. This is surprising since it is generally believed that ecosystems functioning, stability and efficiency are linked to diversity (e.g., Loreau et al., 2001). Moreover, the few existing studies on marine phytoplankton communities assessed seasonal to pluriannual periods (Venrick et al., 1997; Caroppo et al., 1999; Gómez and Gorsky, 2003). Short-time scale studies of the phytoplankton diversity dynamics (i.e. day to day or more often) have concerned lakes (Padisák et al., 1988; Padisák, 1993; Pinckney et al., 1998) or were based on pigment analyses or automated submerged flow cytometer in seawater systems (Bidigare and Ondrusek, 1996; Thyssen et al., 2008). Short term dynamics of marine phytoplankton abundance and diversity are then poorly documented even if phytoplankton could be considered as a good indicator for quick environmental changes (see review in Thyssen et al., 2008).

In order to study the impact of natural disturbances (as wind events and changes in the mixing depth) on marine microphytoplankton abundance and taxonomic diversity as well as related dynamics of main microzooplanktonic groups, we

Published by Copernicus Publications on behalf of the European Geosciences Union. 
analysed samples taken during 4 periods of 5 days from the cruise DYNAPROC 2 (DYNAmic of rapid PROCesses in water column that control vertical flux of matter) performed in the NW Mediterranean from September to November 2004. This expected transition period from summertime oligotrophic stratified system to autumnal system was considered favourable and pertinent because of higher frequencies of wind events. It was completing the DYNAPROC 1 cruise conducted in May 1995 in order to document the effects of episodic wind events on the dynamics of the ecosystem during a post-bloom period, characterised by the transition from a mesotrophic to an oligotrophic system (Andersen and Prieur, 2000). We also analyzed the intake of taxonomical studies in biogeochemical cycle's studies, mass indicators or trophic aspects. This was feasible since DYNAPROC 2 was a multidisciplinary cruise that documented many hydrological parameters and considerable stocks and rates measurements (Andersen et al., 2009).

\section{Material and method}

\subsection{Cruise track}

The DYNAPROC 2 cruise was conducted in 2004 from 14 September to 17 October (Julian days 261 to 292) on board R/V Thalassa, at the vicinity of the NW Mediterranean French JGOFS Station DYFAMED $\left(43^{\circ} 25^{\prime} \mathrm{N}, 7^{\circ} 52^{\prime} \mathrm{E}\right)$, approximately $45 \mathrm{~km}$ South of Cape Ferrat, France, where maximal depth is $2350 \mathrm{~m}$. It was divided into two legs (here after leg 1 and leg 2) that consisted of two cycles of five days each (cycles 1 to 4). In order to describe the hydrological environment, a grid of 16 stations, centred on a fixed station ("central point"), was occupied at least four times during the cruise. All net and water sampling were done near the central point during the whole cruise. Many hydrological parameters are then available since the whole project consisted in evaluating the DYNAmic of rapid PROCesses in water column that control vertical flux of matter. In order to quantify the stability of the water column, an index of the intensity of the stratification was calculated as suggested by Bowden (1983) as: $\Phi=\mathrm{g} / H \int_{-H}^{0}(\rho \mathrm{m}-\rho) \mathrm{zdz}$, where $\Phi$ is an index of potential energy anomaly $\left(\mathrm{J} \mathrm{m}^{-3}\right), H$ is the water column height (m), $\rho$ is the density at any depth $z$, and $\rho \mathrm{m}$ is the mean density of the water column. This index estimates the deficit in potential energy due to a density gradient. A highly mixed water column will present small values of $\Phi$. Water density was derived from temperature-salinity measurements. Integrated values of $\Phi$ were obtained for the 0-90 m layer for all stations. More explanations on cruise development and hydrological/meteorological evolutions could be found in Andersen et al. (2009).

\subsection{Field sampling}

Two complementary sampling methods were used to sample the main microplankton groups, in order to obtain the most exhaustive and accurate description of the species possible, with emphasis put on phytoplanktonic groups. Hydrological bottle sampling allowed collecting small-sized and abundant microphytoplanktonic species. This method was also appropriate to preserve delicate or colonial organisms. Net sampling was the most appropriate to efficiently collect less abundant species (which were often the biggest) since the net filtered a great volume of seawater. Dominant groups of the microzooplankton were also enumerated.

\section{Hydrological bottle sampling}

Samples were collected every $10 \mathrm{~m}$ from $90 \mathrm{~m}$ to the surface with hydrological NISKIN bottles coupled with a Seabird CTD (Conductivity-Temperature-Depth) instrument that recorded continuously hydrological parameters (temperature, salinity, density and in situ fluorescence). Aliquots of $250 \mathrm{ml}$ of seawater from each depth, fixed with acid lugol's solution (final concentration, 2\% v/v; Throndsen, $1978)$ were stored in dark cold room $\left(4^{\circ} \mathrm{C}\right)$ until microscopic analysis. Samples were collected every day of each cycle ( $\sim 12.00$ a.m., ITU), yielding a total of 200 samples. Aliquots were analysed using the Utermöhl method with sedimentation of $100 \mathrm{ml}$ seawater in settling chambers. Counts and observations were done with an inverted light microscope (Zeiss@Axiovert 35), at 200x and 400x magnification. For each sample, a minimum of 250 living cells was counted. Instead of analysing strictly microphytoplankton species, which are formally defined as ranging from 20 to $200 \mu \mathrm{m}$, we preferred counting every species belonging to the 3 groups typical of the microphytoplankton: diatoms, dinoflagellates and silicoflagellates. For instance, some small species of the genus Gymnodinium, with a mean size lower than $10 \mu \mathrm{m}$, were counted. Coccolithophorids were not counted, since acid lugol's solution alters calcified structures. Enumeration of microzooplanktonic groups (tintinnids and naked ciliates) was also carried out. Naked ciliates were separated in 3 size classes (equivalent diameter $<30 \mu \mathrm{m}$, between 30 and $50 \mu \mathrm{m}$, $>50 \mu \mathrm{m})$.

\section{Net sampling}

In addition to the hydrological bottle sampling, we also made daily net sampling during each cycle using a phytoplankton net ( $53 \mu \mathrm{m}$ mesh size, $54 \mathrm{~cm}$ diameter and $280 \mathrm{~cm}$ length). A vertical haul was performed from $90 \mathrm{~m}$ depth to the surface at the same time of the day ( $\sim 10.00$ a.m., ITU) to collect large microplankton. The volume filtered by the net was calculated as equivalent to the surface at the opening of the net multiplied by the height of sampled water column. Due to bad 
weather, there were no net samples for Julian days 285 and 289. The samples were split once or twice using a Motoda splitter (Motoda, 1959) and preserved with acid lugol's solution (Throndsen, 1978) to $2 \%$ final concentration. The cells were enumerated in $1 \mathrm{~mL}$ calibrated slides using a light microscope (Alphaphot 2-YS2, Nikon Instruments, Badhoevedorp, Netherlands). More than 250 living cells were counted per samples. Dinoflagellates were identified at the genus level. We focussed more particularly on genus Ceratium Schrank since it is specious in the Mediterranean and often dominates among large armoured dinoflagellates, thus constituting a good model for biodiversity studies (Tunin-Ley et al., 2007). Determination of taxa belonging to genus Ceratium was carried out at intraspecific level. Although they do not belong to phytoplankton, the total radiolarians were also enumerated in net samples, without detailed taxonomic identification, because they exhibited significant abundances.

\subsection{Estimation of taxonomic diversity}

\subsubsection{Taxonomic identification}

The species identification of cells within the 3 selected groups was based on the following references: Trégouboff and Rose (1957a, b), Massuti and Margalef (1960), Dodge (1982), Sournia (1986), Ricard (1987), Balech (1988), Hasle and Syversten (1996), Steidinger and Tangen (1997) and Horner (2002). In net samples, Ceratium cells were identified at the infraspecific level, using the monograph and the parasystematic nomenclature published by Sournia (1967). Recent molecular and morphological analysis assigned all marine Ceratium species to Neoceratium gen. nov. (Gómez et al., 2010). This new genus name was not used in this work since this naming is still in discussion among specialists (Calado and Huisman, 2010). Moreover, it is rarely used by scientists working on ecology or diversity of microphytoplankton. Some limits with regard to identification constrained our results. Thus, for small-sized cells, resolution of light microscopy and fixation with acid lugol made some determinations difficult. For instance, some Gymnodiniales (dinoflagellates) determination was completed to the genus level. However, different species were distinguished via size, form and ornamentation. In the same way, species name were not ascribed to some Pseudo-Nitzschia, which were discriminated as several species belonging to Pseudo-nitzchia delicatissima complex or Pseudo-nitzchia seriata complex (Hasle and Syversten, 1996).

\subsubsection{Diversity indexes}

Biodiversity parameters were calculated for the main planktonic groups in bottle samples on one hand, and in net samples on the other hand. The taxonomic richness $S$ corresponds to the number of taxa in each group. The diversity was calculated using Shannon's index $\mathrm{H}^{\prime}=-\sum \mathrm{pi} \log _{2} \mathrm{pi}$, where $\mathrm{pi}=\mathrm{ni} / \mathrm{N}, \mathrm{ni}=$ number of individuals of one taxon and $\mathrm{N}=$ total number of individuals. Evenness was estimated as Pielou's index $\mathbf{J}^{\prime}=\mathrm{H}^{\prime} / \log _{2} \mathrm{~S}$. The net samples were divided into three replicates before counting. This allowed to apply the nonparametric jackknife 1 method (Manly, 1991), which gives estimations of the biodiversity parameters after correcting the bias induced by the sampling effort and the sampling size (Carpentier and Lepêtre, 1999). For richness, jackknife $1=\mathrm{SO}+(\mathrm{r} 1(\mathrm{n}-1) / \mathrm{n})$, where $\mathrm{SO}$ is the observed taxonomic richness, $\mathrm{n}$ the number of replicates and $\mathrm{r} 1$ the number of taxa occurring in one single replicate. For diversity and evenness, jackknife $1=\Sigma \Phi \mathrm{i} / \mathrm{n}$, with $\Phi \mathrm{i}=\mathrm{nSt}-(\mathrm{n}-1) \mathrm{Sti}-1$, where $\mathrm{St}$ is the estimation of the indicator for the $\mathrm{n}$ replicates and Sti-1 the estimation of the indicator for the $n-1$ replicates. These estimations were calculated on a minimal basis of 100 counted cells per replicate, which represents a minimal total of 300 cells per sample. For diversity calculations, Magurran (2004) recommended to use abundances ranging from 200 to 500 cells per samples. The jackknife 1 method was not applied on bottle samples, for which no replicates were available. In order to identify the important genus or taxa within dinoflagellates and within genus Ceratium from net samples, a dominance index was also calculated as $\delta=$ $100(n 1+n 2) / N$, where $n 1$ and $n 2$ are the abundances of the two most important taxa and $\mathrm{N}$ the total cell concentration (Hulburt, 1963).

\subsection{Data analysis}

The variability of the main planktonic groups was analysed using a multivariate analysis producing a synthesis of cooccurring factors. The Correspondence Analysis (CA) aims to describe the total inertia of a multidimensional set of data in a sample of fewer dimensions (or axes) that is the best summary of the information contained in the data (Benzecri, 1973). The CA is applied to contingency tables and uses a Chi square metric. The different calculations of this method are detailed in Legendre and Legendre (2000). This ordination method was applied to our matrix describing the planktonic groups in bottle samples (samples in rows and planktonic groups in columns), in order to underline the kinetics of plankton composition over time. The composite mean depths' and the composite mean day's coordinates, calculated as the average of the samples of the same depth, or the average of the samples of the same day, were used as illustrative qualitative variables.

Spearman's rank coefficients (Siegel and Castellan 1988) were used in order to explore the correlations between planktonic groups diversity and the stratification index, as estimation of a disturbance. 


\section{Results}

\subsection{Physical environment}

\subsubsection{Meteorological data}

Wind regime was characterised by the alternation of strong wind events and calm periods. The leg 1 was accompanied with two significant wind events (Fig. 1a), one at the beginning of cycle 1 (day 261 to day 262), which lasted $48 \mathrm{~h}$, and a latter one during cycle 2 (day 269) with a highest intensity yet shorter in time. Both corresponded to NE winds, whereas winds are usually blowing from $\mathrm{W}$ or SW in the area at this period (Raybaud et al., 2008). By contrast, the end of leg 2 was dominated by persistent and strong $\mathrm{NE}$ and $\mathrm{SW}$ winds resulting in a decrease of stratification (Fig. 4a). In addition, an episode with significant precipitations occurred just before the campaign started.

\subsubsection{Hydrological and biological parameters (CTD acquisition)}

The hydrological structure along the main part of the cruise revealed a typical late summer situation with a stratified water column, whereas a transition from summer period to autumn structure was expected (i.e. destratification). Thus, the thermocline ranged from 20 to $40 \mathrm{~m}$ depth (Fig. 1b), with surface temperatures above $20^{\circ} \mathrm{C}$. Salinity dynamics were characterised by the intrusion of Low Salinity Water (LSW) with likely coastal characteristics $(<38.3)$ during leg 1 (day 265 to day 274) just under the thermocline (Fig. 1c). This phenomenon, detailed by Andersen et al. (2009) and Raybaud et al. (2008), was also observed to a lesser degree during cycle 4 (day 283 to day 286). Each time, this resulted in a light shallowing of the thermocline, as a consequence of the dilatation of the less dense water mass. Along with a deepening of the temperature and the halocline, the destratification seemed to be initiated at the end of the cruise. The significant decrease of the stratification index observed at this period (Fig. 4a) depicted the disturbance that occurred in response to the regime of persistent and strong wind at this time. At the beginning of cycle 1 , the fluorescence measurements displayed a chlorophyll- $a$ peak about $70-80 \mathrm{~m}$ depth, followed by another distinctive one about $50 \mathrm{~m}$ depth (Fig. 1d). All along the second part of the cruise, the Deep Chlorophyll Maximum (DCM) was located just under the thermocline, following its shift to a deeper depth. Consequently, the in-vivo fluorescence evolution showed up tendency to get deeper at the end of campaign.

\subsection{Abundance and diversity of microplankton}

\subsubsection{Abundances}

Summing over the whole cruise, 151 species of microphytoplankton were found in hydrological bottle samples. The

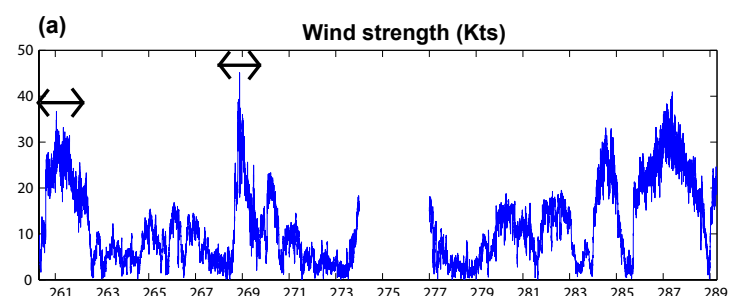

(b) Water temperature $\left({ }^{\circ} \mathrm{C}\right)$

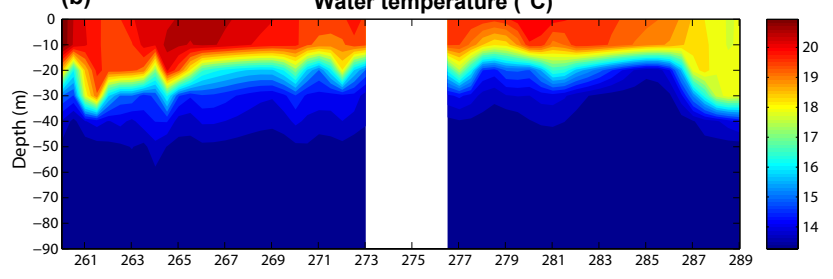

(c) Salinity

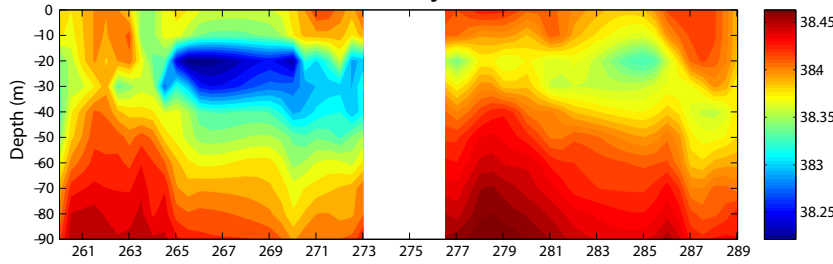

(d) Relative fluorescence

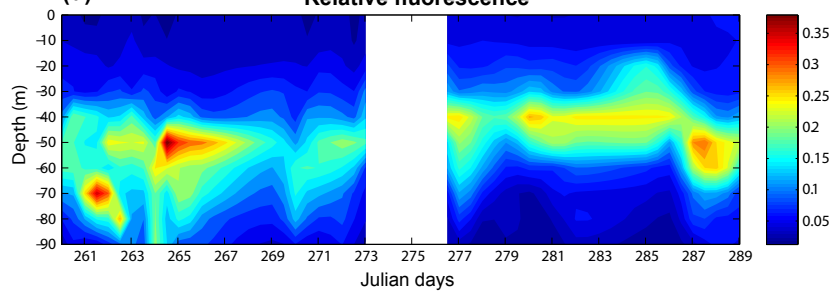

Fig. 1. Hydrological conditions during DYNAPROC 2 cruise, (a) Wind strength, (b) Water temperature, (c) Salinity, (d) Relative fluorescence.

most diverse group was dinoflagellate, with 92 species (32 armoured and 60 unarmoured), followed by 55 species of diatoms ( 35 centrics and 25 pennates). We found only 2 species of silicoflagellates. Additionally we encountered Leptocylindrus mediterraneus cells (centric diatom), but they were integrated neither in abundance nor in diversity calculation since they were not alive. Near 58\% of taxa were determined to the species level, $98 \%$ to genus level; the rest was composed of indeterminate armoured dinoflagellates. Maximum abundances for each taxa over the whole cruise period and the whole water column were indicated in Table 1 . The maximum abundance of total microphytoplankton was often just under the thermocline and reached more than 40000 ind $\mathrm{l}^{-1}$ at the beginning of cycle 4 (Fig. 2a). Throughout the study, diatoms were mostly in the lower depths (Fig. 2b), with abundance ranging from 200 to 800 cell $1^{-1}$ in surface and from 1000 up to 5000 cell $^{-1}$ below the thermocline. Temporally, two distinct peaks of abundance were observed. The first one (from 4000 to 5000 cell $^{-1}$ ) occurred at $80 \mathrm{~m}$ depth at the beginning of the first cycle (Julian day 262) and was 
Table 1. List of microphytoplanktonic species encountered in hydrological bottle samples during DYNAPROC 2 cruise, with maximal abundance recorded in samples in cells per liter.

\begin{tabular}{|c|c|c|}
\hline Species & Authorities & Max. abundance \\
\hline \multicolumn{3}{|l|}{ Bacillariophyceae } \\
\hline Achnanthes sp. a & Bory & 60 \\
\hline Bacteriastrum delicatulum & Cleve & 100 \\
\hline Bacteriastrum furcatum & Shadbolt & 60 \\
\hline Cerataulina dentata & Hasle & 180 \\
\hline Cerataulina pelagica & (Cleve) Hendey & 20 \\
\hline Chaetoceros affinis & Lauder & 60 \\
\hline Chaetoceros decipiens & Cleve & 60 \\
\hline Chaetoceros didymus & Ehrenberg & 80 \\
\hline Chaetoceros laciniosus & Schütt & 400 \\
\hline Chaetoceros peruvianus & Brightwell & 80 \\
\hline Chaetoceros sp. a & Ehrenberg & 40 \\
\hline Chaetoceros sp. b & Ehrenberg & 240 \\
\hline Climacosphenia sp. a & Ehrenberg & 120 \\
\hline Corethron sp. & Castracane & 3240 \\
\hline Coscinodiscus sp. a & Ehrenberg & 120 \\
\hline Coscinodiscus sp. b & Ehrenberg & 20 \\
\hline Cylindrotheca closterium & (Ehrenberg) Reimann \& Lewin & 180 \\
\hline Dactyliosolen phuketensis & (Sundström) Hasle & 600 \\
\hline Entomoneis sp. a & Ehrenberg & 20 \\
\hline Gossleriella tropica & Shütt & 40 \\
\hline Guinardia striata & (Stolterfoth) Hasle & 200 \\
\hline Haslea wawrikae & (Husedt) Simonsen & 140 \\
\hline Hemiaulus hauckii & Grunow ex Van Heurck & 10 \\
\hline Hemidiscus cuneiformis & Wallich & 20 \\
\hline Lauderia annulata & Cleve & 150 \\
\hline Leptocylindrus danicus & Cleve & 140 \\
\hline Leptocylindrus minimus & Gran & 240 \\
\hline Lyrella sp. a & Karajeva & 20 \\
\hline Navicula sp. a & Bory & 50 \\
\hline Navicula sp. b & Bory & 40 \\
\hline Navicula sp. c & Bory & 40 \\
\hline Navicula sp. d & Bory & 2800 \\
\hline Nitzschia bicapitata & Cleve & 670 \\
\hline Planktonielle sol & (Wallich) Schütt & 30 \\
\hline Pleurosigma sp. a & Smith & 20 \\
\hline Proboscia alata & (Brightwell) Sundström & 160 \\
\hline Pseudo-nitzschia sp. a (Nitzschia delicatissima complex) & Peragallo & 760 \\
\hline Pseudo-nitzschia sp. b (Nitzschia delicatissima complex) & Peragallo & 1300 \\
\hline Pseudo-nitzschia sp. c (Nitzschia seriata complex) & Peragallo & 800 \\
\hline Pseudo-nitzschia sp. d (Nitzschia seriata complex) & Peragallo & 900 \\
\hline Pseudo-nitzschia sp. e (Nitzschia seriata complex) & Peragallo & 360 \\
\hline Pseudo-nitzschia sp. f (Nitzschia seriata complex) & Peragallo & 380 \\
\hline Pseudo-nitzschia sp. g (Nitzschia seriata complex) & Peragallo & 100 \\
\hline Rhizosolenia decipiens & Sundström & 60 \\
\hline Rhizosolenia hebetata f. semispina & (Hensen) Gran & 40 \\
\hline Synedra pulchella & Kützing & 160 \\
\hline Synedra sp. a & Ehrenberg & 20 \\
\hline Thalassionema frauenfeldii & (Grunow) Hallegraeff & 280 \\
\hline Thalassionema nitzschioides & (Grunow) Mereschkowsky & 580 \\
\hline Thalassionema sp. a & Grunow ex Mereschkowsky & 740 \\
\hline Thalassiosira sp. a & Cleve & 260 \\
\hline Thalassiosira sp. b & Cleve & 240 \\
\hline Thalassiosira sp. c & Cleve & 380 \\
\hline Thalassiosira sp. d & Cleve & 1200 \\
\hline Toxarium undulatum & Bailey & 20 \\
\hline
\end{tabular}


Table 1. Continued.

\begin{tabular}{|c|c|c|}
\hline \multicolumn{3}{|l|}{ Dinophyceae } \\
\hline Armoured species & & \\
\hline Alexandrium sp. a & Halim & 1200 \\
\hline Alexandrium sp. b & Halim & 800 \\
\hline Amylax triachanta & (Jörgensen) Sournia & 20 \\
\hline Ceratium declinatum & (Karsten) Jörgensen & 20 \\
\hline Ceratium furca & (Ehrenberg) Claparède \& Lachmann & 20 \\
\hline Ceratium fusus & (Ehrenberg) Dujardin & 20 \\
\hline Ceratium hexacanthum & Gourret & 20 \\
\hline Ceratium horridum & (Cleve) Gran & 20 \\
\hline Ceratium limulus & Gourret & 10 \\
\hline Ceratium lineatum & (Ehrenberg) Cleve & 20 \\
\hline Ceratium pentagonum & Gourret & 50 \\
\hline Ceratium platycorne & Daday & 20 \\
\hline Ceratocorys sp. a & Stein & 40 \\
\hline Dinophysis acuminata & Clarapède \& Lachmann & 20 \\
\hline Dinophysis acuta & Ehrenberg & 20 \\
\hline Dinophysis dens & Pavillard & 10 \\
\hline Dinophysis hastata & Stein & 20 \\
\hline Dinophysis pulchella & (Lebour) Balech & 60 \\
\hline Dinophysis punctata & Jörgensen & 40 \\
\hline Dinophysis sphaerica & Stein & 40 \\
\hline Diplopsalis sp. a & Bergh & 2400 \\
\hline Gonyaulax digitale & (Pouchet) Kofoid & 20 \\
\hline Gonyaulax polygramma & Stein & 20 \\
\hline Gonyaulax sp. a & Diesing & 20 \\
\hline Gonyaulax spinifera & (Claparède \& Lachmann) Diesing & 40 \\
\hline Heterocapsa rotundata & (Lohmann) Hansen & 1800 \\
\hline Heterodinium leiorhynchum & (Murray \& Whitting) Kofoid & 80 \\
\hline Heterodinium sp. a & Kofoid & 20 \\
\hline Heterodinium sp. b & Kofoid & 20 \\
\hline Heterodinium sp. b & Kofoid & 20 \\
\hline Katodinium glaucum & (Lebour) Loeblich III & 4400 \\
\hline Lingulodinium polyedrum & (Stein) Dodge & 40 \\
\hline Oblea sp. a & Balech ex Loeblich \& Loeblich & 70 \\
\hline Oxytoxum constrictum & (Stein) Bütschli & 20 \\
\hline Oxytoxum laticeps & Schiller & 40 \\
\hline Oxytoxum longiceps & Schiller & 40 \\
\hline Oxytoxum milneri & Murray \& Whitting & 20 \\
\hline Oxytoxum reticulatum & (Stein) Schütt & 40 \\
\hline Oxytoxum scolopax & Stein & 40 \\
\hline Oxytoxum tesselatum & Stein & 50 \\
\hline Pavillardinium sp. a & De Toni & 40 \\
\hline Phalacroma rapa & Jorgensen & 10 \\
\hline Phalacroma rotundatum & (Claparède \& Lachmann) Kofoid \& Michener & 60 \\
\hline Phalacroma sp. a & Stein & 20 \\
\hline Podolampas palmipes & Stein & 40 \\
\hline Podolampas spinifera & Okamura & 60 \\
\hline Prorocentrum gracile & Schütt & 510 \\
\hline Prorocentrum scutellum & Schröder & 20 \\
\hline Prorocentrum triestinum & Schiller & 300 \\
\hline Protoperidinium cassum & Balech (Balech) & 300 \\
\hline Protoperidinium divergens & (Ehrenberg) Balech & 20 \\
\hline Protoperidinium ovatum & Pouchet & 20 \\
\hline Protoperidinium pyriforme & (Paulsen) Balech & 20 \\
\hline Protoperidinium sp. a & Bergh & 800 \\
\hline Schuettiella mitra & (Schütt) Balech & 460 \\
\hline Scrippsiella sp. a & (Stein) Loeblich III & 12800 \\
\hline
\end{tabular}


Table 1. Continued.

\begin{tabular}{|c|c|c|}
\hline Spiraulax kofoidii & Graham & 40 \\
\hline Tryblionella compressa & (Bailey) Poulin & 280 \\
\hline Undeterminated armoured Dinoflagellate sp. a & & 40 \\
\hline Undeterminated armoured Dinoflagellate sp. b & & 20 \\
\hline Undeterminated armoured Dinoflagellate sp. c & & 20 \\
\hline \multicolumn{3}{|l|}{ Naked species } \\
\hline Amphidinium sp. a & Claparède \& Lachmann & 4000 \\
\hline Amphidinium sp. b & Claparède \& Lachmann & 4020 \\
\hline Asterodinium gracile & Sournia & 20 \\
\hline Cochlodinium sp. a & Schütt & 360 \\
\hline Dissodinium pseudolunula & Swift ex Elbrächter \& Drebes & 20 \\
\hline Gymnodinium sp. a & Stein & 20000 \\
\hline Gymnodinium sp. b & Stein & 800 \\
\hline Gymnodinium sp.c & Stein & 5600 \\
\hline Gymnodinium sp. d & Stein & 8000 \\
\hline Gymnodinium sp. e & Stein & 160 \\
\hline Gymnodinium sp. $\mathrm{f}$ & Stein & 2400 \\
\hline Gymnodinium sp. g & Stein & 20 \\
\hline Gymnodinium sp. h & Stein & 60 \\
\hline Gymnodinium sp. i & Stein & 2400 \\
\hline Gymnodinium sp. j & Stein & 16800 \\
\hline Gymnodinium sp. k & Stein & 20 \\
\hline Gymnodinium sp. 1 & Stein & 60 \\
\hline Gyrodinium falcatum & Kofoid \& Swezy & 20 \\
\hline Gyrodinium sp. a & Kofoid \& Swezy & 7200 \\
\hline Gyrodinium sp. b & Kofoid \& Swezy & 8800 \\
\hline Gyrodinium sp. c & Kofoid \& Swezy & 2800 \\
\hline Gyrodinium sp. d & Kofoid \& Swezy & 3500 \\
\hline Gyrodinium sp. e & Kofoid \& Swezy & 1340 \\
\hline Gyrodinium sp. f & Kofoid \& Swezy & 1400 \\
\hline Gyrodinium sp. g & Kofoid \& Swezy & 2000 \\
\hline Gyrodinium sp. h & Kofoid \& Swezy & 1200 \\
\hline Gyrodinium sp. i & Kofoid \& Swezy & 20 \\
\hline Kofoidinium velelloides & Pavillard & 100 \\
\hline Petalodinium porcelio & Cachon \& Cachon & 60 \\
\hline Polykrikos kofoidii & Chatton & 80 \\
\hline Pronoctiluca pelagica & Fabre-Domergue & 160 \\
\hline Scaphodinium mirabile & Margalef & 20 \\
\hline Torodinium teredo & (Pouchet) Kofoid \& Swezy & 140 \\
\hline \multicolumn{3}{|l|}{ Dictyochophyceae } \\
\hline Dictyocha fibula & Ehrenberg & 420 \\
\hline Dictyocha speculum & Ehrenberg & 750 \\
\hline
\end{tabular}

mainly the centric species Corethron sp. probably in senescent phase since intracellular part was sometimes affected. The second peak occurred at $50 \mathrm{~m}$ depth during cycle $3(\mathrm{Ju}-$ lian day 278) and was mainly composed of pennate species represented by the complexes Pseudo-nitzchia delicatissima spp. and Pseudo-nitzchia seriata spp. In samples of cycles 1 and 2, we also found the centric species Leptocylindrus mediterraneus and its epiphyte (Fig. 3a), the heterotrophic flagellate Rhizomonas setigera.
In contrast to the diatoms, dinoflagellates showed a preference for the upper layers during the whole cruise (Fig. 2c). The group of naked dinoflagellates, mainly represented by small-sized species Gyrodinium spp. and Gymnodinium spp., dominated in both legs. Abundance increased from 5000 to

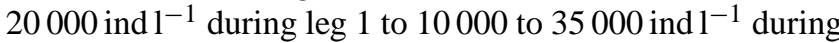
cyles 3 and 4. The observed armoured species Scrippsiella sp. was probably associated with the intrusions of less saline water (Fig. 3b). Silicoflagellates (Dictyochophyceae) abundance was lowest in comparison with the two other groups 
Table 2. List of dinoflagellates genera and Ceratium taxa encountered in net samples during DYNAPROC 2 cruise, with maximal abundance recorded in samples in cells per liter.

\begin{tabular}{|c|c|c|c|}
\hline Dinoflagellates & Max. abundance & Genus Ceratium & Max. abundance \\
\hline Acanthogonyaulax (Kofoid) Graham & 6 & C. arietinum Cleve var. arietinum & 10 \\
\hline Amphidiniopsis Woloszynska & 3 & C. arietinum var. gracilentum (Jørgensen) Sournia & 3 \\
\hline Amphisolenia Stein & 8 & C. arietinum Cleve "arietinum-gracilentum" & 71 \\
\hline Centrodinium Kofoid & 11 & C. azoricum Cleve & 7 \\
\hline Ceratium Schrank & 3734 & C. candelabrum (Ehrenberg) Stein var. candelabrum & 61 \\
\hline Ceratocorys Stein & 105 & C. candelabrum var. depressum (Pouchet) Jørgensen & 70 \\
\hline Corythodinium Loeblich Jr. \& Loeblich III & 134 & C. candelabrum (Ehrenberg) Stein "candelabrum-depressum" & 30 \\
\hline Dinophysis Ehrenberg & 237 & C. candelabrum(Ehrenberg) Stein "candelabrum-depressum" & 24 \\
\hline Dissodinium Klebs in Pascher & 36 & C. candelabrum (Ehrenberg) Stein "depressum-candelabrum" & 35 \\
\hline Goniodoma Stein & 1420 & C. carriense Gourret var. carriense & 16 \\
\hline Gonyaulax Diesing & 465 & C. carriense var. volans (Cleve) Jørgensen & 18 \\
\hline Heterocapsa Stein & 26 & C. carriense Gourret "carriense-volans" & 3 \\
\hline Heterodinium Kofoid & 30 & C. concilians Jørgensen & 237 \\
\hline Kofoidinium Pavillard & 81 & C. contrarium (Gourret) Pavillard & 71 \\
\hline Micracanthodinium Deflandre & 3 & C. declinatum f. brachiatum Jørgensen & 145 \\
\hline Noctiluca Suriray & 21 & C. declinatum f. normale Jørgensen & 145 \\
\hline Ornithocercus Stein & 16 & C. declinatum var. majus Jørgensen & 30 \\
\hline Oxytoxum Stein & 60 & С. eиarcuatum Jørgensen & 26 \\
\hline Palaeophalacroma Schiller & 7 & C. extensum (Gourret) Cleve & 65 \\
\hline Phalacroma Stein & 346 & C. falcatiforme Jørgensen & 35 \\
\hline Podolampas Stein & 289 & C. falcatum (Kofoid) Jørgensen & 16 \\
\hline Prorocentrum Ehrenberg & 17 & C. furca var. eugrammum (Ehrenberg) Schiller & 59 \\
\hline Protoperidinium Bergh & 342 & C. furca (Ehrenberg) Clarapède \& Lachmann "furca-eugrammum" & 4 \\
\hline Pyrophacus Stein & 42 & C. furca (Ehrenberg) Clarapède \& Lachmann "furca-eugrammum" & 59 \\
\hline Pyrocystis Murray ex Haeckel & 29 & C. furca (Ehrenberg) Clarapède \& Lachmann "eugrammum-furca" & 14 \\
\hline Spatulodinium Cachon \& Cachon & 53 & C. fusus (Ehrenberg) Dujardin & 149 \\
\hline \multirow[t]{2}{*}{ Spiraulax Kofoid } & 79 & C. gibberum var. dispar (Pouchet) Sournia & 19 \\
\hline & & C. gravidum Gourret & 7 \\
\hline \multirow[t]{37}{*}{ No genera: 27} & & C. hexacanthum f. hiemale Pavillard & 4 \\
\hline & & C. hexacanthum Gourret var. hexacanthum & 5 \\
\hline & & C. horridum var. buceros (Zacharias) Sournia & 13 \\
\hline & & C. horridum (Cleve) Gran "horridum-buceros" & 14 \\
\hline & & C. horridum (Cleve) Gran "buceros-horridum" & 298 \\
\hline & & C. inflatum (Kofoid) Jørgensen & 4 \\
\hline & & C. limulus (Gourret ex Pouchet) Gourret & 219 \\
\hline & & C. longirostrum Gourret & 30 \\
\hline & & C. longissimum (Schröder) Kofoid & 4 \\
\hline & & C. macroceros (Ehrenberg) Vanhöffen var. macroceros & 32 \\
\hline & & C. macroceros (Ehrenberg) Vanhöffen "macroceros-gallicum" & 24 \\
\hline & & C. massiliense f. armatum (Karsten) Jørgensen & 5 \\
\hline & & C. massiliense (Gourret) Jørgensen var. massiliense & 11 \\
\hline & & C. massiliense var. protuberans (Karsten) Jørgensen & 32 \\
\hline & & C. minutum Jørgensen & 11 \\
\hline & & C. paradoxides Cleve & 3 \\
\hline & & C. pavillardii Jørgensen & 12 \\
\hline & & C. pentagonum f. turgidum (Jørgensen) Jørgensen & 80 \\
\hline & & C. pentagonum var. tenerum Jørgensen & 316 \\
\hline & & C. pentagonum Gourret "robustum-tenerum" & 62 \\
\hline & & C. pentagonum Gourret "robustum-tenerum" & 1587 \\
\hline & & C. platycorne Daday var.platycorne & 96 \\
\hline & & C. platycorne var.compressum (Gran) Jørgensen & 267 \\
\hline & & C. platycorne Daday "platycorne-compressum" & 56 \\
\hline & & C. platycorne Daday "compressum-platycorne" & 99 \\
\hline & & C. ranipes Cleve & 22 \\
\hline & & C. schroeteri Schröder & 3 \\
\hline & & C. setaceum Jørgensen & 24 \\
\hline & & C. symmetricum Pavillard var. symmetricum & 113 \\
\hline & & C. symmetricum var. coarctatum (Pavillard) Graham \& Bronikovsky & 151 \\
\hline & & C. symmetricum var. orthoceras (Jørgensen) Graham \& Bronikovsky & 17 \\
\hline & & C. trichoceros (Ehrenberg) Kofoid & 6 \\
\hline & & C. tripos var. atlanticum (Ostenfeld) Paulsen & 83 \\
\hline & & C. tripos var. pulchellum (Schröder) Lopez & 97 \\
\hline & & C. tripos (Müller) Nitzsch "atlanticum-pulchellum" & 9 \\
\hline & & C. tripos (Müller) Nitzsch "atlanticum-pulchellum" & 35 \\
\hline & & $\begin{array}{l}\text { No species: } 34 \\
\text { No infraspecific taxa: } 64\end{array}$ & \\
\hline
\end{tabular}



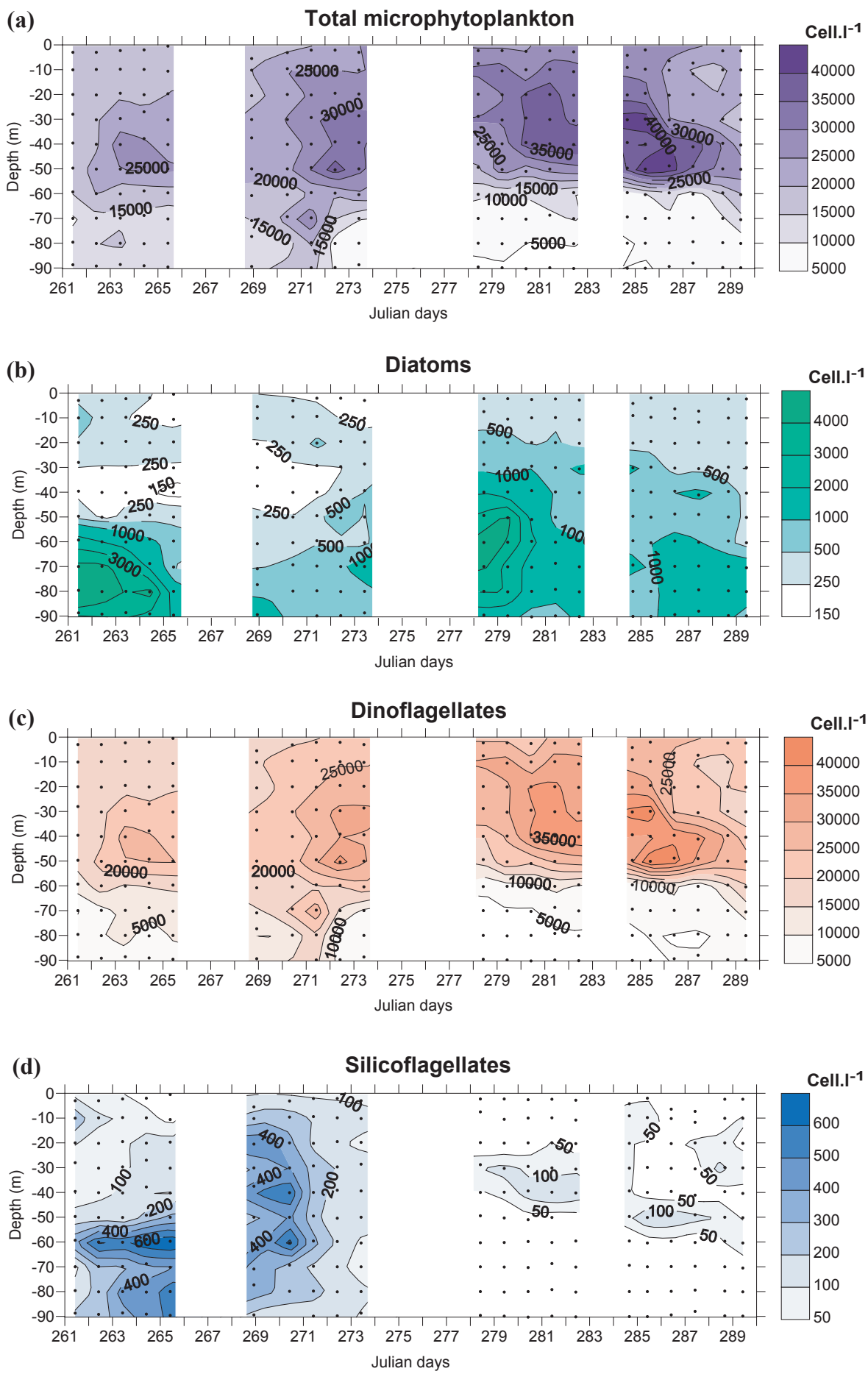

Fig. 2. Abundance of microphytoplankton during DYNAPROC 2 cruise, from hydrological bottle sampling (a) Total microphytoplankton, (b) Bacillariophyceae, (c) Dinophyceae, (d) Dictyochophyceae.

(Fig. 2d). Two species were found, Dictyocha fibula and Dictyocha speculum, the first one being the most abundant. Silicoflagellates showed marked spatio-temporal variations. Abundance varied from less than 50 cell $^{-1}$ to more than 600 cell $1^{-1}$. During the 2 first cycles, two maxima of abun- dance were identifiable (days 264-265 and 270 with a range from 400 cell $^{-1}$ up to 600 cell $^{-1}$ ). The end of cycle 2 marked the decrease of abundance that appeared then really weak during the two last cycles. Finally, the evolution of naked ciliates abundances (Fig. 3c) showed a slight increase 

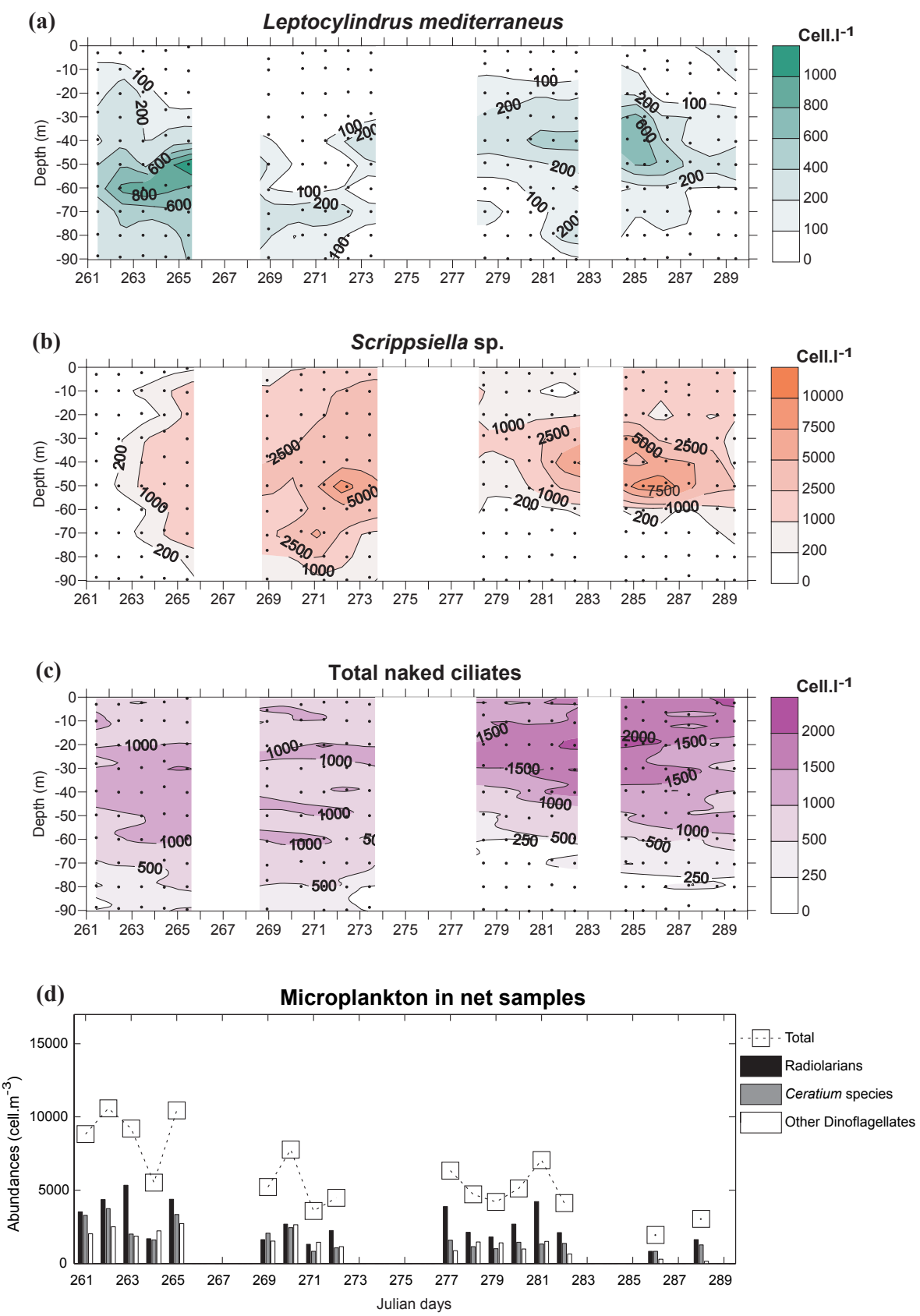

Fig. 3. Abundance of ecologically important planktonic organisms during DYNAPROC 2 cruise, from hydrological bottle sampling (a) The diatom Leptocylindrus mediterraneus, (b) The dinoflagellate Scrippsiella sp., (c) Naked ciliates, and from net sampling (d) Dinoflagellates genera and Ceratium infraspecific taxa.

from 500 to 1250 cell $1^{-1}$ during the first cycles leading to maximal value of 2000 cell $^{-1}$ in cycles 3 and 4 . In each sample, the great majority (more than $90 \%$ ) of ciliates was of small size (less than $30 \mu \mathrm{m}$ ).

Microplankton abundance in net samples, corresponding to radiolarians and large armoured dinoflagellates, decreased throughout the study, from over 8000 cells per $\mathrm{m}^{3}$ at the beginning to about 3000 cells per $\mathrm{m}^{3}$ at the end of the cruise (Fig. 3d). Overall, radiolarians were the dominant group (from 828 to 5344 cell m$^{-3}$ ), while Ceratium spp. represented about $50 \%$ (from 834 to 3734 cell m$^{-3}$ ) of the total dinoflagellates. Ceratium became largely dominant within dinoflagellates at the end of the cruise, while the total microplankton abundance decreased. The maximum abundances of each taxa from net sampling are indicated in Table 2 . 

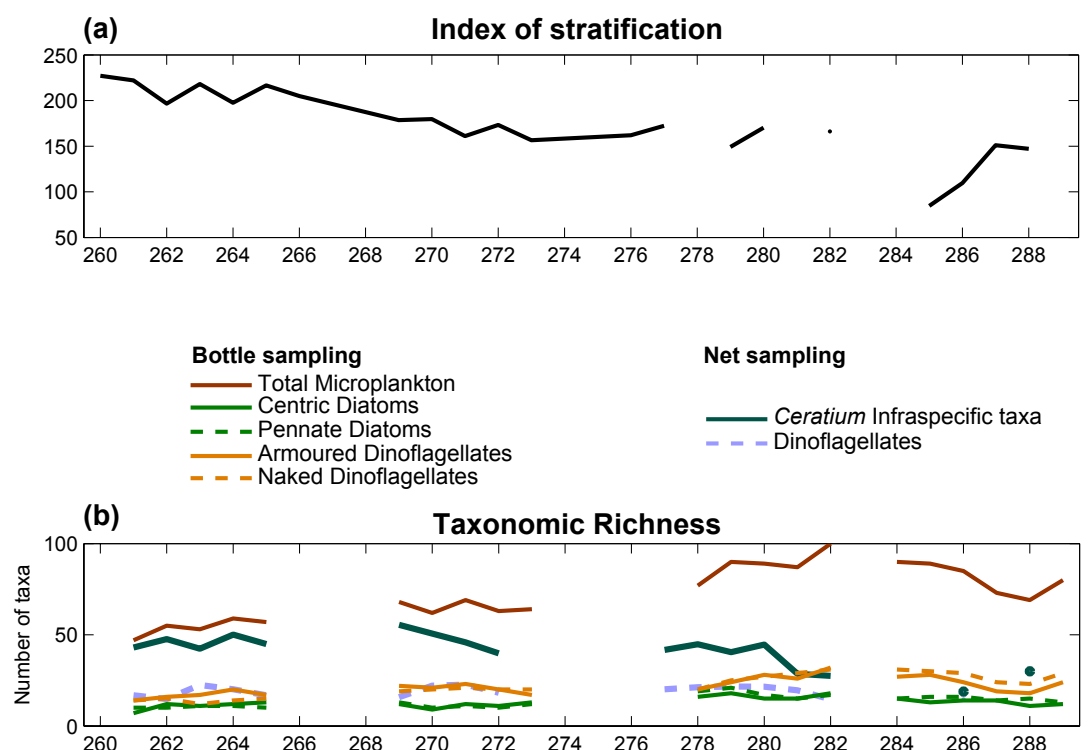

(c)

Diversity Shannon's Index

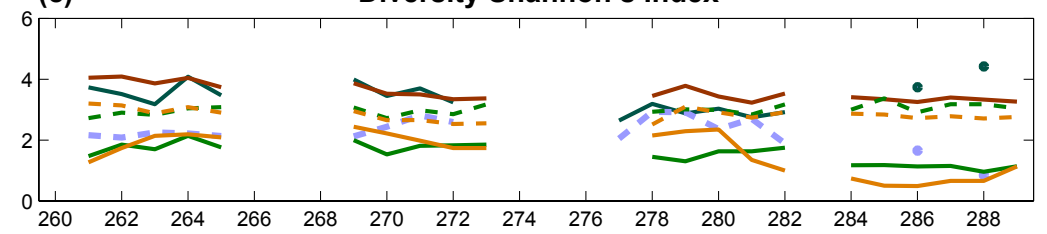

(d) Evenness Pielou's Index

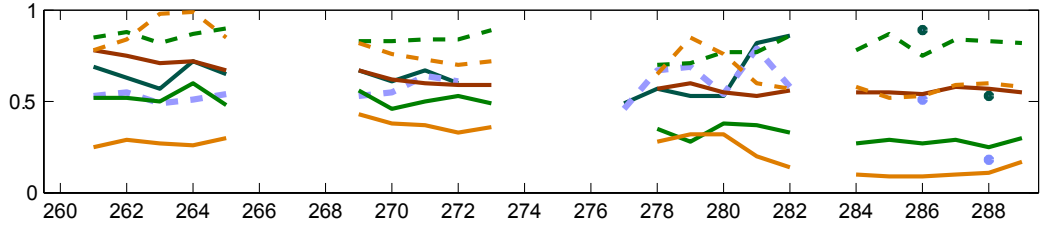

Fig. 4. Evolution of the stratification index in relation to the biodiversity descriptors of the main groups of the microphytoplankton during DYNAPROC 2 cruise, from hydrological bottle and net sampling (a) Stratification index, (b) Taxonomic richness, (c) Diversity Shannon's index, (d) Evenness Pielou's index.

Table 3. Spearman rank correlations between the stratification index $(\Phi)$ and taxonomic richness, diversity Shannon index and evenness Pielou index of the microplankton components.

\begin{tabular}{llll}
\hline & Taxonomic richness S & Diversity Shannon index $\mathrm{H}^{\prime}$ & Evenness Pielou Index $\mathrm{J}^{\prime}$ \\
\hline Microphytoplankton & $-0.82^{* * *}$ & $0.78^{* * *}$ & $0.84^{* * *}$ \\
\hline Diatoms & $-0.73^{* * *}$ & $-0.47^{*}$ & -0.02 \\
Centric diatoms & $-0.53^{*}$ & $0.55^{*}$ & $0.76^{* * *}$ \\
Pennate diatoms & $-0.72^{*}$ & $-0.58^{*}$ & 0.31 \\
Dinoflagellates & $-0.77^{* *}$ & $0.81^{* * *}$ & $0.71^{* * *}$ \\
Armoured dinoflagellates & $-0.57^{*}$ & $0.49^{*}$ & 0.33 \\
Naked dinoflagellates & $-0.85^{* * *}$ & 0.40 & $0.75^{* * *}$ \\
\hline
\end{tabular}

\footnotetext{
* Correlation is significant at the 0.05 level;

** Correlation is significant at the 0.01 level;

$* * *$ Correlation is significant at the 0.001 level.
} 

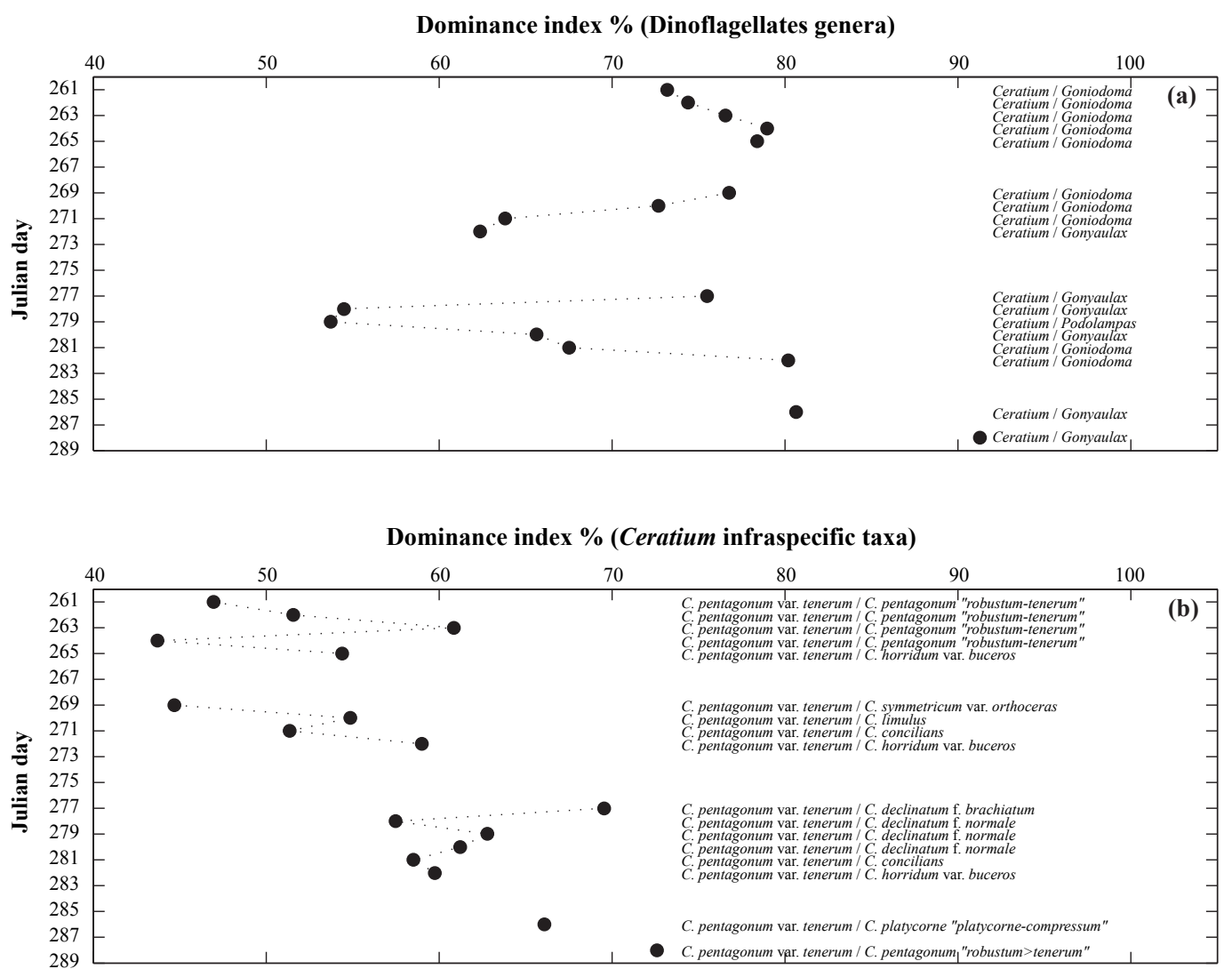

Fig. 5. Evolution of the dominance index in net samples (a) Dinoflagellates genera dominance index, (b) Ceratium taxa dominance index.

\subsubsection{Diversity patterns}

The total richness of the microphytoplankton from hydrological bottle samples varied from 47 to 100 species (Fig. 4b). The total richness slightly increased during the first cycles, reached a maximum during the cycle 3 and then decreased during cycle 4 although values stayed superior to the richness observed in leg 1. Diatoms and dinoflagellates respective richness followed the same pattern than total microphytoplankton, but the increase was more marked for dinoflagellates richness. Number of diatoms species ranged from 17 to 39 , with nearly equal portions of pennates (10 to 21 species) and centrics (7 to 18 species) (Fig. 4b). Dinoflagellate species richness ranged from 28 to 63 species, with a similar contribution of armoured species (14 to 32 species) and naked species (12 to 31 species).

In net samples, while a total of 27 dinoflagellates genera was described (Table 2), the generic richness in dinoflagellates was about 20 genera along the study (Fig. 4b). Within the genus Ceratium, the number of infraspecific taxa increased from 43 to 56 taxa during the cycle 1 and then decreased after day 269 to reach a value of 28 taxa.

The diversity of microphytoplankton in bottle samples was about 3-4 bit ind ${ }^{-1}$ and was globally constant (Fig. 4c). Diversity indexes for total dinoflagellates and total diatoms showed the same feature. Yet, there was a trend of decreasing diversity of armoured species within dinoflagellates and of the diversity of centric species within diatoms (Fig. 4c). Actually, the few variations in diversity resulted in opposite trends in richness and evenness indexes. Indeed, the evenness index sensibly decreased for total microphytoplankton (Fig. 4d), and was mainly driven by a pattern of decreasing dinoflagellates evenness, both displayed by armoured species and by naked species to a lesser extent. In contrast, the evenness of total diatoms remained at a high level without any clear trend of variation. Evenness of pennate diatoms was constant whereas it slowly decreased for centric species (Fig. 4d). Thus, while overall diversity appeared to be quite invariant, important variations occurred among specific taxonomic groups. Exploring the relationship between biodiversity and the stratification index, we were able to highlight a trend: among each microplanktonic communities, the taxonomic richness was negatively related to the stratification index (Table 3). We observed higher diversity indexes of dinoflagellates and centric diatoms associated to stratified waters (higher $\Phi$ ) while total diatoms and particularly pennate group diversity was negatively correlated to the stratification index (Spearman coefficient $R_{s}=-0.58, P<0.05$; Table 3). 
In net samples, the evenness varied few during the cycles 1 and 2 for dinoflagellates, but became more variable during the latter cycles with a final decrease that followed a trend to increase. Within the genus Ceratium, infraspecific diversity slowly decreased and then consequently increased at the end of the cruise (Fig. 4c), whereas evenness strongly increased during the cycle 2 and then decreased as for dinoflagellates genera at the very end of the last cycle (Fig. 4d). Regarding to the relationship between diversity in net samples and the index of stratification, the correlations found were not significant.

In term of dominance, the genus Ceratium was prominent all along the cruise within the dinoflagellates, and was associated to the genus Goniodoma during the first leg, then alternatively to the genera Goniodoma, Gonyaulax and Podolampas (Fig. 5a). The association Ceratium/Podolampas at the middle of cycle 3 corresponded to a minimum of the dominance index (about 50\%) whereas during the last cycle, the dominance of the association Ceratium/Gonyaulax exceeded $90 \%$.

Within the genus Ceratium, the dominance index tended to increase throughout the study from $44 \%$ to a maximum of $73 \%$ at the end, and was globally higher during the 2 last cycles (Fig. 5b). One single taxon, C. pentagonum var. tenerum, corresponding to the thermophilic variety of the species $C$. pentagonum, also dominated all along the cruise. It was associated to $C$. pentagonum "robustumtenerum”, C. horridum var. buceros, C. symmetricum var. orthoceras, $C$. limulus and $C$. concilians during the cycles 1 and 2 , and to $C$. declinatum f. brachiatum, C. declinatum f. normale, C. concilians, C. horridum var. buceros, C. platycorne "platycorne-compressum" and C. pentagonum "robustum>tenerum" during the cycles 3 and 4 . These 10 prominent taxa represented a few part of the 64 infraspecific taxa which were encountered in the genus Ceratium (Table 2).

\subsection{Spatio-temporal dynamics of microplankton}

The Correspondences Analysis, applied to the abundances of the main planktonic groups, yielded an interesting synthesis of the spatio-temporal patterns of the microplankton (Fig. 6). The planktonic groups were discriminated by their different coordinates on the 2 first factors, explaining respectively $54.8 \%$ and $24.3 \%$ of the total inertia. Thus, siliceous organisms, corresponding to centric and pennate diatoms and silicoflagellates, had negative coordinates on axis 1 , while armoured dinoflagellates and the mainly mixotrophic groups, composed of ciliates and naked dinoflagellates, had coordinates close to 0 on this axis. The axis 2 discriminated centric diatoms, Dictyochophyceae and armoured dinoflagellates (negative coordinates) from pennate diatoms and the mainly heterotrophic group (positive coordinates). Projection of sampling days and sampling depths as illustrative variables indicated that the planktonic groups had particu-

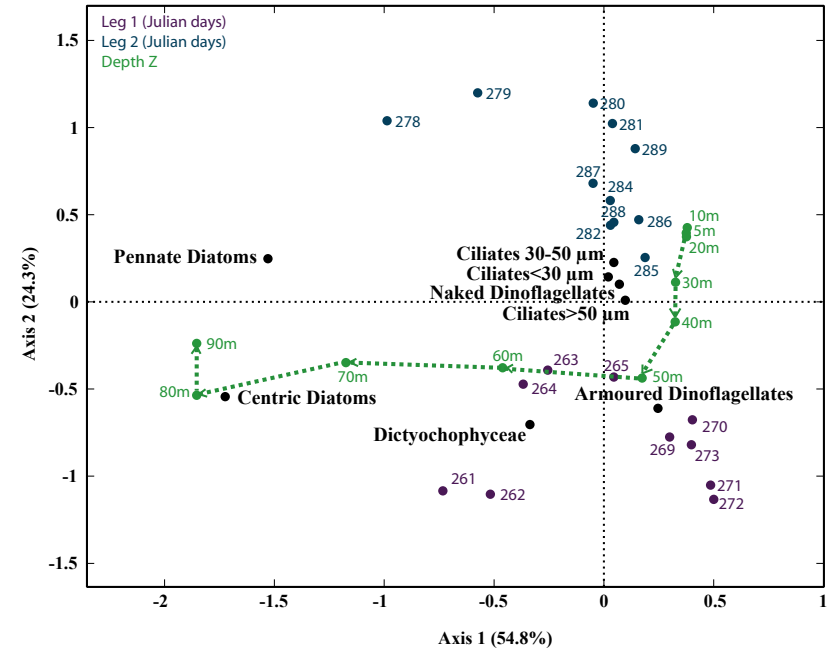

Fig. 6. Spatial and temporal dynamics of the microplankton from hydrological bottle sampling during DYNAPROC 2 cruise. The Correspondence Analysis was applied on the main microplanktonic groups. Sampling depths and Julian days were added as illustrative variables (the coordinates were calculated as the average of samples of the same days or of the same depth).

lar vertical distribution: the mixotrophic group with ciliates and naked dinoflagellates was located above the thermocline in the surface layer; the armoured dinoflagellates were associated with depths close to $50 \mathrm{~m}$, i.e. close to the mean depth of the thermocline and the chlorophyll maximum; Dictyochophyceae and more particularly diatoms were characteristic of the deepest layers. In addition, there was an opposition on axis 2 between the days of the cycles 1 and 2, associated with diatoms, Dictyochophyceae and armoured dinoflagellates, and the days of the cycles 3 and 4 associated with ciliates, naked dinoflagellates and pennate diatoms.

\section{Discussion}

\subsection{Dynamics of microplankton}

In the NW Mediterranean Sea, the summer period usually starts in May-June with the development of the thermocline that lasts until mid-September or late October, when storms frequency is high. Along with a reinjection of nutrients from depth toward upper layers, as a result of mixing effect of the storms and wind events, the development of a high pressure system following the storm period leads to calm and sunny weather conditions, known as St Martin's summer, which triggers the autumnal phytoplankton bloom in the NW Mediterranean (Duarte et al., 1999). Despite the specific choice in timing for the DYNAPROC 2 cruise, the conditions that we encountered seemed to be characteristic of late summer stratification, with a well-established thermocline and a situation of nutrient depletion in the surface 
layer (Marty et al., 2008). Although a first wind episode initiated the destratification, the settlement of a mixing system was only obvious at the end of the campaign in response to persistent winds during the cycle 4 . The dominance of small-sized microplankton species such as ciliates, mixotrophic and heterotrophic dinoflagellates (mainly Gyrodinium spp. and Gymnodinium spp.) and radiolarians also corresponded to a typical summer situation. Dinoflagellates usually dominate the microphytoplankton during the stratified period in the NW Mediterranean (Gómez, 2003; Gómez and Gorsky, 2003), since they do prefer conditions of low turbulence and warm temperatures, while low nutrients concentrations in the surface layer and stratification limit the development of diatoms and silicoflagellates. The significant contribution of naked dinoflagellates probably resulted from a better adaptation to an oligotrophic environment, since several species are known to have phagotrophic activity (Steidinger and Tangen, 1997; Stoecker, 1999). Within less abundant armoured dinoflagellates, the prominence of Ceratium species, which characterised the net samples, has been already reported in the Ligurian Sea (Halim, 1960; Gómez and Gorsky, 2003). The present abundances are of the same magnitude, even slightly lower, than those observed in the coastal waters of Villefranche bay at the same time (Tunin-Ley et al., 2007). Although the majority of Ceratium species possesses chloroplasts, the cells can contain vacuoles, which could result from phagotrophic behaviour (Chang and Carpenter, 1994). Radiolarians also significantly develop in late summer and autumn (Lange et al., 1997). Within radiolarians, the polycystines occur in the upper strata of the water column as they may bear algal symbionts, with maximal abundances ranging from 25 to $50 \mathrm{~m}$ depth in extrapolar areas (Anderson et al., 2000). Observed densities were remarkably higher than those usually observed, with an order of magnitude of tens to hundreds individuals per cubic meter. During the first DYNAPROC cruise (May 1995), abundances of radiolarians ranged from 200 to more than $1000 \mathrm{ind} \mathrm{m}^{-3}$ with maximal abundances comprised between 50 and $70 \mathrm{~m}$ (Andersen et al., 2001), that represents slightly lower abundances than those reported for the present cruise. Radiolarians may have significantly contributed to primary production since it has been established their association with photosynthetic symbionts (Foster et al., 2006). The naked ciliates represented an important part of the total microplankton, especially during the leg 2 (day 269 to day 273) and their abundances were consistent with values described in the Villefranche bay at the same period (Gómez and Gorsky, 2003).

In addition to the overall dominance of small-sized dinoflagellates during the study, we observed a temporal shift in microplankton composition from a system characterised by the presence of autotrophic organisms and by the significant contribution of siliceous species (leg 1: day 261 to day 273) to a regeneration-dominated system with mainly mixotrophic or heterotrophic organisms during leg 2 (day 276 to 291). The slight decrease in silicate concentration found during leg 2 (Marty et al., 2008) may partly explain the decline of siliceous organisms. Moreover, the development in the deep layer of diatoms, species that are able to respond very fast to nutrient pulses (Cloern and Dufford, 2005), was clearly associated to local increases in silicate concentrations (Marty et al., 2008). The disappearance of the silicoflagellates could then result then from the competition with diatoms. As the first observed population of diatoms dominated by Corethron sp. was senescent, the competition for silica was weak, likely allowing the development of the silicoflagellates, whereas the competition for silica may have increased towards the middle of the campaign with the significant development of mainly pennate diatoms. Predation pressure also differed along the study, with a change in zooplankton species composition (Raybaud et al., 2008). Both diatoms (which were represented by several large-sized species), and silicoflagellates (which are potentially protected from herbivorous copepod predation due to their large spins) were well represented during leg 1 (Julian day 259 to 274). Their contribution decreased during leg 2 , corresponding with the presence of other predator species with more generalist and non-selective diets like Pteropods (Raybaud et al., 2008).

The trophic importance of microzooplankton is well recognized; nevertheless, this group is often exclusively composed of a ciliate "box" in food web studies and models. We assumed that the contribution of hetero- and mixotrophic dinoflagellates is largely underestimated. Since Lessard and Swift $(1985,1986)$, we know that about half of dinoflagellates species in marine plankton do not possess chloroplasts and likely consume other plankton cells. A review of Sherr and Sherr (2007) showed that dinoflagellate biomass could sometimes be higher than ciliate biomass while small naked dinoflagellates may account for a large fraction (up to 90\%) of the total heterotrophic dinoflagellates biomass. This last point was confirmed in Pacific Ocean by Masquelier and Vaulot (2007) who found, using epifluorescence microscopy, that about $80 \%$ of heterotrophic dinoflagellates were cells smaller than $20 \mu \mathrm{m}$ and noted that typical Utermöhl method largely underestimated small dinoflagellates abundances. A large number of dinoflagellates species recorded during our study were mixotrophic or heterotrophic. For instance, all Protoperidinium species are known to lack photosynthetic pigments. Numerous cells of the order Gymnodiniales were observed, but our preserving and counting methods probably largely underestimated their abundance (Masquelier and Vaulot, 2007). Given the abundance and variety of ciliates and heterotrophic dinoflagellates, the ecosystem of our study had a heterotrophic microplankton pool that feed on a wide range of prey, from bacteria to centric diatoms (Sherr and Sherr, 2007). The heterotrophic microplankton likely had another important role, that of a significant food resource for mesozooplankton (Perez et al., 1997; Susuki et al., 1999; Liu et al., 2005). This has to be taken into consideration when studying the whole ecosystem functioning. 
The dynamics of marine phytoplankton have been assessed over short scales using pigment analysis (Vidussi et al., 2000). HPLC technique based on the pigment signature of major phytoplankton groups constitutes an efficient tool to have an overview of relative contributions and major shifts. This technique has been used successfully during DYNAPROC 2 (Marty et al., 2008). Additionally, converting pigments to carbon equivalent could be convenient for linking the overall distribution of phytoplankton to the dynamic of organic matter. Yet, with this approach, groups of species that have no specific pigment signature are not identified, as well as groups without any photosynthetic pigments (i.e. heterotrophic dinoflagellates). Furthermore, species composition cannot be considered. Compared to pigment diversity analysis our direct microscopic observations supply complementary information of biogeochemical and ecological interests. Thus, silicoflagellates characterised by a complex pigment-content composed of acylfucoxanthines (19'-BF et 19'-HF), fuco-, diadino-, diato-xanthine and also chlorophylle $a, c$, and $c 3$ (Daugbjerg and Henriksen, 2001) can not be monitored using a single pigment determination method but are easily distinguished microscopically. During DYNAPROC 2, the dynamics of silicate compounds does not only concern diatoms; the siliceous silicoflagellates, even though less abundant, participated in both silicate and carbon fluxes. The abundance of Dictyocha spp. encountered during the first period of the cruise, argues for a non-negligible contribution in the silicate cycle and the existence of competition with diatoms for the nutrient resources, especially silicate. This aspect is highlighted during the first low salinity event that may have constituted a physiological stress with a stronger impact on diatoms. Meanwhile silicate was available; it precisely corresponded with the highest abundances of silicoflagellates. Assuming that turbulences increased the velocity of the phytoplankton cells sinking (Ruiz et al., 2004) and that this velocity also depends on the cell shape, the specific modification that occurred within the community probably influenced geochemical processes. In addition to silicate fluxes, our study also provides information on the nitrogen cycle. The peculiar consortium constituted by frustule of Leptocylindrus mediterraneus and the protozoan Rhizomonas setigera (Pavillard) Patterson, is involved in nitrogen $\left(\mathrm{N}_{2}\right)$ fixation. As the individuals observed in oligotrophic Pacific Ocean (Gómez, 2007), R. setigera was always found restricted to the central section of the frustules. Indeed, this aplastial protist is characterised by a symbiosis with a nitrogen-fixing cyanobacterium (Buck and Bentham, 1998). The dynamics of $L$. mediterraneus may be important with regard to nitrogen budgets. Its increase in abundance noted during the second part of cruise may have helped to sustain the primary production.

\subsection{Biodiversity and physical perturbations}

The changes described in the microplankton community should be related to the changes observed in the environment. This potentially raises two questions. First, did the physical perturbations that occurred (i.e. wind stress and low salinity water upcoming) affect the biodiversity of microplankton? Second, do our data support the Intermediate Disturbance Hypothesis (IDH)? Indeed, according to Connell (1978), an intermediate level of disturbance leads to a peak of diversity. In the absence of disturbance, the competitive exclusion principle (Hardin, 1960) would apply, the less-fit species being eliminated, resulting in low diversity. In contrast, highly intense or frequent disturbance can restrict the community to a few pioneer species, yielding low diversity. This theory, firstly developed for terrestrial plants, has been largely tested on phytoplankton, in part as an attempt to solve the "paradox of the plankton" (Hutchinson, 1961), that points out the incoherence between the remarkable high richness observed in phytoplankton and the few resources which theoretically prevents the coexistence of numerous species, because of the principle of competitive exclusion. According to previous laboratory and enclosure experiments as well as field studies, mainly focussing on freshwater ecosystems, the IDH appears to be applicable to phytoplankton (Padisák, 1994; Sommer, 1995; Flöder and Sommer, 1999; Hambright and Zohary, 2000; Elliott et al., 2001; Weithoff et al., 2001; Shea et al., 2004). But the weakness of this theory is that it considers the response to an unmeasured event (Reynolds, 1995). While it appears effectively difficult to define what a disturbance is and how intense it is, it has been proposed to consider a disturbance an event that alters the niche opportunities available to the species in a system (Shea et al., 2004).

In our study, the stratification index, being the resultant of physical perturbations (wind regime, intrusion of low salinity water), can be considered as a proxy of disturbance. Thus, exploring putative correlations between physical disturbances and biodiversity of phytoplankton from hydrological bottles, we observed that the decrease of the evenness and the increase of the specific richness were associated to the decrease of the stratification index. Despite the intrusion of less saline water and the on-going destratification that initiated during the first leg, the diversity of the total microplankton from bottle samples was quite constant along the study but the richness increased. Although the horizontal advection was weak, the less saline water mass has brought new species, as illustrating by the development of the coastal Scrippsiella sp., or additional individuals of some already present species, modifying the relative abundances of the species and consequently the diversity. We can postulate that perturbations were weak and progressive along the study, leading to a consistent total microplankton richness accompanied with coexistence of species at the end of the cruise when the destratification was likely established. Weak and frequent disturbances (several moderate wind events) would 
have favoured and sustained diversity (here, namely species richness).

On the opposite, in net samples, the biodiversity followed a different evolution, with a remarkable increase in Ceratium species dominance within dinoflagellates. Even within the genus Ceratium, which is known to be an interesting model for biodiversity studies (Tunin-Ley et al., 2007), the evenness tended to decrease at the very end of the cruise, reflecting an increase in the dominance of a couple of taxa. This reverse evolution may illustrate the lower abilities of some dinoflagellates, with low growth rates as in Ceratium taxa, to adapt to changes occurring at short-time scale. We can postulate that those non-opportunistic species can not get benefit from the gradual disturbance which favoured the diversity of other groups.

\subsection{Indicator and particular species}

Species-specific analysis also permits special attention to indicator species. The dinoflagellate Scrippsiella $\mathrm{sp}$. is associated with the less saline water incoming during the first and the second leg of the cruise. As the species belonging to this genus are considered as neritic (Hasle and Syvertsen, 1996), we thus provide evidence for the coastal origin of the water with this biological marker. The species of this genus was not determined, even if it was morphologically close to Scrippsiella trochoidea, since Montresor et al. (2003) have suggested the existence of cryptic species in the Mediterranean Sea. Moreover, several cells of the naked dinoflagellate Asterodinium gracile, with five radiating elongated extensions as presented in Fig. 2a in Gómez and Claustre (2003), were sampled in our study (one cell at $50 \mathrm{~m}$ and the other at $60 \mathrm{~m}$ depth, Julian day 286). This species was initially reported in Indian Ocean (Sournia, 1986), later in the eastern Mediterranean Sea (Abboud-Abi Saab, 1989) and Gómez and Claustre (2003) found this species in the Tyrrhenian basin considering it as a possible biological indicator of warming. To our knowledge, it is the first time that $A$. gracile was found in the Ligurian Sea and this could indicate an extension of the spatial distribution of this species. Nevertheless, some publications suggest that the genus Asterodinium may correspond to life stages of a highly versatile single species regrouping taxa of the genera Brachydinium, Karenia and $\mathrm{Mi}$ croceratium (Gómez et al., 2005; Gómez, 2006).

Despite their large size, some dinoflagellate species characterized by non-typical or much modified morphologies are often underestimated or absent from most of microphytoplankton analysis. According to Gómez and Furuya (2004), this could be explained by a specific and not easily accessible literature and difficulty in the identification, due to folding in fixed cells. Careful analysis of our samples revealed several modified dinoflagellates. They mainly belonged to the family Leptodiscaceae Taylor (order Noctilucales Heackel). The species are strongly antero-posteriorly flattened and display a wing-like extension, called the velum. Two speci- mens of Scaphodinium mirabile were found in sub-surface (10 $\mathrm{m}$ depth, Julian day 280 and $2 \mathrm{~m}$ depth, Julian day 285). This species, considered as thermophylic (Stoyanova 1999; Gómez and Furaya, 2004), has already been found in NW Mediterranean Sea (Margalef, 1963; Léger, 1971; Gómez and Gorsky, 2003). We encountered 18 specimens of Petalodinium porcelio, between 10 and $90 \mathrm{~m}$ depth (Julian days 279 to 289). It is the first time that this species has been recorded in Mediterranean Sea since its description in 1969 (Cachon and Cachon, 1969). The two other cells of P. porcelio were reported by Stoyanova (1999) in the Black Sea and by Gómez and Furuya (2005) in the Pacific Ocean. The most relative abundant morphologically-modified dinoflagellate was $\mathrm{Ko}$ foidinium velelloides, which belongs to the family Kofoidiniaceae Taylor (Sournia, 1986). Seventy-five specimens were found, from surface to $90 \mathrm{~m}$ depth, throughout the study period. This species has a complex life cycle and we assumed that specimens of immature stage resembling Gymnodinium belong to this species, due to the morphology of the large velum at mature sampled stages. The species of the genus is not easy to identify because of the high morphological variability during the life cycle and the difficulties to delimit the species from preserved specimens (Gómez and Furaya, 2007). As for genus Asterodinium (see above), those rare species need more investigation about description as well as biology and ecology.

\section{Conclusions}

This is one of the first detailed studies dealing with daily variations of marine microplankton abundance and diversity, done with two complementary sampling methods (i.e. hydrological bottles and plankton net). Our results highlighted the value of such data to complete pigments analysis; the knowledge of microplanktonic short-term abundance and diversity evolution supplied complementary information of biogeochemical, biological and ecological interests. It allowed a better understanding of the interactions between autotrophs and nutrients as well as trophic relations with zooplankton. Finally, indicators species could also confirm coastal water intrusion and potential warming of Mediterranean Sea.

This demonstrates that traditional taxonomic studies are definitely not obsolete, with regard to the recent advances in automatic digitization and classification (Benfield et al., 2007) that will considerably decrease the time allocated to microplankton identification in further studies.

Acknowledgements. We thank the chief scientist, V. Andersen, for organising the DYNAPROC 2 cruise, and the crew of the $\mathrm{R} / \mathrm{V}$ Thalassa for ship operations. We are also grateful to John Dolan for his advices and kind re-lecture. This study was part of the PECHE project; financial support was provided by the I.N.S.U.-C.N.R.S. through the PROOF program (JGOFS-France) and by the project "La Notion d'Espèce dans le Phytoplancton" in the framework of the national program EC2CO "Ecosphère 
Continentale et Côtière". This work was also supported by the ANR AQUAPARADOX and by the MarBEF European Network of Excellence "Marine Biodiversity and Ecosystem Functioning" in the framework of the program MARPLAN for European integration of marine microplankton research. Ph.D. scholarship of A. Tunin-Ley was provided by "Ministère de l'Education Nationale, de l'Enseignement Supérieur et de la Recherche". We thank the anonymous reviewers for their valuable remarks and advices that greatly improved our manuscript. This paper is dedicated to our friend and colleague Valérie Andersen who prematurely passed away in March 2007.

Edited by: E. Marañón

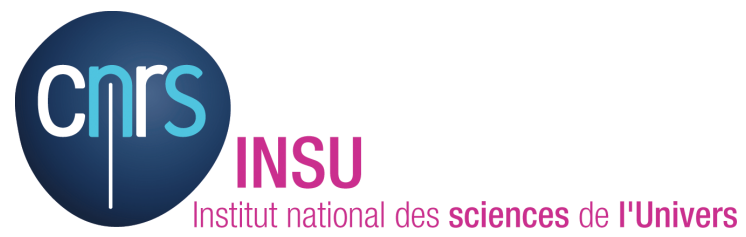

The publication of this article is financed by CNRS-INSU.

\section{References}

Abboud-Abi Saab, M.: Les Dinoflagellés des eaux côtières libanaises-Espèces rares ou nouvelles du phytoplancton marin, Leba. Sci. Bull., 5, 5-16, 1989.

Andersen, V. and Prieur, L.: One-month study in the open NW Mediterranean Sea (DYNAPROC experiment, May 1995): overview of the hydrobiogeochemical structures and effects of wind events, Deep Sea Res. Pt. I, 47, 397-422, 2000.

Anderson, O. R., Nigrini, C., Boltovskoy, D., Takahashi, K., and Swanberg, N. R.: Class Polycystinea, edited by: Lee, J. J., Leedale, G. F., Bradbury, P., The illustrated guide to Protozoa, Second edition, Society of Protozoologists, Lawrence, KA, USA, 994-1022, 2000.

Balech, E.: Los dinoflagelados del Atlantico sudoccidental, Ph.D. Thesis, Instituto Español de Oceanografia, Madrid, Espagne, 1988.

Benfield, C. M., Grosjean, P., Culverhouse, P. F., Irigoin, X., Sieracki, M. E., Lopez-Urrutia, A., Dam, H. G., Hu, Q., Davis, C. S., Hansen, A., Pilskaln, C. H., Riseman, E. M., Schultz, H., Utgoff, P. E., and Gorsky, G.: RAPID : research on automated plankton identification, Oceanography, 20, 172-187, 2007.

Benzecri, J. P.: L'analyse des données. II. L'analyse des correspondances, Dunod, Paris, 619 pp., 1973.

Bidigare, R. R. and Ondrusek, M. E.: Spatial and temporal variability of phytoplankton pigment distributions in the central equatorial Pacific Ocean, Deep Sea Research Part II: Topical Studies in Oceanography, 43, 809-833, 1996.

Bowden, K. F: Physical oceanography of coastal waters, Ellis Horwood Series on Marine Science, John Wiley \& Sons, New York, 302 pp, 1983.

Buck, K. R. and Bentham, W. N.: A novel symbiosis between a cyanobacterium, Synechococcus sp., an aplastidic protist, Solenicola setigera, and a diatom, Leptocylindrus mediterraneus, in the open ocean, Mar. Biol., 132, 349-355, 1998.
Cachon, J. and Cachon, M.: Contribution à l'étude des Noctilucidae Saville-Kent. Evolution, morphologie, cytologie, systématique. II. Les leptodiscinae Cachon J. et M., Protistologica, 5, 11-33, 1969.

Calado, A. J. and Huisman, J. M.: Commentary: Gómez, F., Moreira, D., and Lopez-Garcia, P.: Neoceratium gen. nov., a New Genus for All Marine Species Currently Assigned to Ceratium (Dinophyceae), Protist, 161, 517-519, 2010.

Carpentier, P. and Lepêtre, A.: Robustesse de quelques indices de diversité à l'échantillonnage, Océanis, 25, 435-455, 1999.

Caroppo, C., Fioccab, A., Sammarcob, P., and Magazzu'b, G.: Seasonal Variations of Nutrients and Phytoplankton in the Coastal SW Adriatic Sea (1995-1997), Botanica Marina, 42, 389-400, 1999.

Chang, J. and Carpenter, E. J.: Active growth of the oceanic dinoflagellate Ceratium teres in the Caribbean and Sargasso seas estimated by cell cycle analysis, J. Phycol., 30, 375-381, 1994.

Cloern, J. E. and Dufford, R.: Phytoplankton community ecology: principles applied in San Francisco Bay, Mar. Ecol. Prog. Ser., 285, 11-28, 2005.

Connell, J. H.: Diversity in Tropical Rain Forests and Coral Reefs, Science, 199, 1302-1310, 1978.

Daugbjerg, N. and Henriksen, P.: Pigment composition and RbcL sequence data from the Silicoflagellate Dictyocha speculum: a heterokont alga with pigments similar to some haptophytes, J. Phycol., 37, 11-10, 2001.

Dodge, J. D.: Marine Dinoflagellates of the British Isles, Her Majesty's Stationery Office, England, 303 pp, 1982.

Duarte, C. M., Agustí, S., Kennedy, H., and Vaqué, D.: The Mediterranean climate as a template for Mediterranean marine ecosystems: the example of the northeast Spanish littoral, Prog. Oceanogr., 44, 245-270, 1999.

Elliott, J. A., Irish, A. E., and Reynolds, C. S.: The effects of vertical mixing on a phytoplankton community: a modelling approach to the intermediate disturbance hypothesis, Fresh. Biol., 46, 12911297, 2001.

Falkowski, P. G., Katz, M. E., Knoll, A. H., Quigg, A., Raven, J. A., Schofield, O., and Taylor, F. J. R.: The Evolution of Modern Eukaryotic Phytoplankton, Science, 305, 354-360, 2004.

Field, C. B., Behrenfeld, M. J., Randerson, J. T., and Falkowski, P. G.: Primary production of the biosphere: Integration terrestrial and oceanic components, Science, 281, 237-240, 1998.

Flöder, S. and Sommer, U.: Diversity in planktonic communities: an experimental test of the intermediate disturbance hypothesis, Limnol. Oceanogr., 44, 1114-1119, 1999.

Foster, R. A., Carpenter, J. E., and Bergman, B.: Unicellular cyanobionts in open ocean Dinoflagellates, Radiolarians, and Tintinnids: ultrastructural characterization and Immunolocalization of phycoerythrin and nitrogenase, J. Phycol., 42, 453-463, 2006.

Gómez, F. and Claustre, H.: The genus Asterodinium (Dinophyceae) as possible biological indicator of warming in the western Mediterranean Sea, J. Mar. Biol. Ass., UK, 83, 173-174, 2003.

Gómez, F. and Furaya, F.: New records of Scaphodinium mirabile (Dinophyceae), an unnoticed dinoflagellate in the Pacific Ocean, Phycol. Res., 52, 13-16, 2004.

Gómez, F. and Furaya, F.: Leptodiscaceans (Noctilucales, Dinophyceae) from the Pacific Ocean: First records of Petalodinium 
and Leptodiscus beyond the Mediterranean Sea, Europ. J. Protist., 41, 231-239, 2005.

Gómez, F. and Furaya, F.: Kofoidinium, Spatulodinium and other kofoidiniaceans (Noctilucales, Dinophyceae) in the Pacific Ocean, Europ. J. Protist., 43, 115-124, 2007.

Gómez, F. and Gorsky, G.: Annual microplankton cycles in Villefranche Bay, Ligurian Sea, NW Mediterranean, J. Plank. Res., 25, 323-339, 2003.

Gómez, F., Moreira, D., and Lopez-Garcia, P.: Neoceratium gen. nov., a New Genus for All Marine Species Currently Assigned to Ceratium (Dinophyceae), Protist, 161, 35-54, 2010.

Gómez, F., Nagahama, Y., Takayama, H., and Furuya, K.: Is Karenia a synonym of Asterodinium-Brachidinium (Gymnodiniales, Dinophyceae)? Acta Bot. Croat., 64, 263-274, 2005.

Gómez, F.: The Dinoflagellate Genera Brachidinium, Asterodinium, Microceratium and Karenia in the Open SE Pacific Ocean, Algae, 21, 445-452, 2006.

Hambright, K. D. and Zohary, T.: Phytoplankton species diversity control through competitive exclusion and physical disturbances, Limnol. Oceanogr., 45, 110-122, 2000.

Halim, Y.: Etude quantitative et qualitative du cycle écologique des dinoflagellés dans les eaux de Villefranche-sur-Mer, Ann. Inst. Oceanogr. Monaco., 38, 123-232, 1960.

Hardin, G.: The competitive exclusion principle, Science, 131, 1292-1298, 1960.

Hasle, G. R. and Syvertsen, E. E.: Dinoflagellates, edited by: Tomas, C. R., Identifying Marine Phytoplankton, Academic Press, Inc. San Diego, Calif., 526-527, 1996.

Hulburt, E. M.: The diversity of phytoplanktonic populations in oceanic, coastal, and estuarine regions, J. Mar. Res., 21, 81-93, 1963.

Hutchinson, G. E.: The paradox of the plankton, Am. Nat., 95, 137$145,1961$.

Kyewalyanga, M. S., Naik, R., Hegde, S., Raman, M., Barlow, R., and Roberts, M.: Phytoplankton biomass and primary production in Delagoa Bight Mozambique: Application of remote sensing, Estuar. Coast. Shelf Sci., 74, 429-436, 2007.

Lange, C. B., Weinheimer, A., Reid, M. R. H., and Thunell, R.: Sedimentation patterns of diatoms, radiolarians, and silicoflagellates in Santa Barbara basin, California, CalCOFI Rep., 38, 161-170, 1997.

Legendre, P. and Legendre, L.: Numerical Ecology, 2 Edn. Elsevier Science BV, Amsterdam, 853 pp., 2000.

Lessard, E. J. and Swift, E.: Species-specific grazing rates of heterotrophic dinoflagellates in oceanic waters, measured with a dual-label radioisotope technique, Mar. Biol., 87, 289-296, 1985.

Lessard, E. J. and Swift, E.: Dinoflagellates from the North Atlantic classified as phototrophic or heterotrophic by epifluorescence microscopy, J. Plank Res., 8, 1209-1215, 1986.

Liu, H., Dagg, M. J., and Strom, S.: Grazing by calanoid copepod Neocalanus cristatus on the microbial foodweb in the coastal Gulf of Alaska, J. Plankton Res., 27, 647-662, 2005.

Loreau, M., Naeem, S., Inchausti, P., Bengtsson, J., Grime, J. P., Hector, A., Hooper, A., Huston, M. A., Raffaelli, D., Schmid, B., Tilman, D., and Wardle, D. A.: Biodiversity and ecosystem functioning: current knowledge and future challenges, Science, 294, 804-808, 2001.

Magurran, A. E.: Measuring Biological Diversity, Blackwell Pub- lishing, USA, 256 pp, 2004.

Manly, B. F.: Randomization and Monte Carlo methods in biology, Chapman and Hall, London, 292 pp, 1991.

Margalef, R.: Scaphodinium mirabile nov. gen. nov. sp., un nuevo dinoflagelado del plancton marino, Miscelanea Zool. Barcelona, $1,1-2,1963$.

Marty, J. C. and Chiaverini, J.: Seasonal and interannual variations in phytoplankton production at DYFAMED time-series station, northwestern Mediterranean Sea. Deep-Sea Res. Pt. II, 49(11), 1965-1985, 2002.

Marty, J. C., Garcia, N., and Raimbault, P.: Phytoplankton dynamics and primary production under late summer conditions in the NW Mediterranean Sea, Deep Sea Res. Pt. I, 55(9), 1131-1149, 2008.

Masquelier, S. and Vaulot, D.: Distribution of micro-organisms along a transect in the South-East Pacific Ocean (BIOSOPE cruise) from epifluorescence microscopy, Biogeosciences Discuss., 4, 2667-2697, doi:10.5194/bgd-4-2667-2007, 2007.

Montresor, M., Sgrosso, S., Procaccini, G., and Kooistra, W.: Intraspecific diversity in Scrippsiella trochoidea (Dinophyceae): evidence for cryptic species, Phycologia, 42, 56-70, 2003.

Motoda, S.: Devices of simple plankton apparatus, Mem. Fac. Fish. Hokkaido Univ., 7, 73-94, 1959.

Padisák, J.: The influence of different disturbance frequencies on the species richness, diversity and equitability of phytoplankton in shallow lakes, Hydrobiologia, 249, 135-156, 1993.

Padisák, J.: Identification of relevant time-scales in non-equilibrium community dynamics: conclusions from phytoplankton surveys, New Zealand J. Ecol., 18, 169-176, 1994.

Padisák, J., Tóth, L. G., and Rajczy, M.: The role of storms in the summer succession of the phytoplankton community in a shallow lake (Lake Balaton, Hungary), J. Plank. Res., 10, 249-265, 1988.

Perez, M. T., Dolan, J. R., and Fukai, E.: Planktonic oligotrich ciliates in the NW Mediterranean: growth rates and consumption by copepods, Mar. Ecol. Prog. Ser., 155, 89-101, 1997.

Pinckney, J. L., Paerl, H. W., Harrington, M. B., and Howe, K. E.: Annual cycles of phytoplankton community-structure and bloom dynamics in the Neuse River Estuary, North Carolina, Mar. Biol., 131, 371-381, 1998.

Raybaud, V., Nival, P., Mousseau, L., Gubanova, A., Altukhov, D., Khvorov, S., Ibañez, F., and Andersen, V.: Short term changes in zooplankton community during the summer-autumn transition in the open NW Mediterranean Sea: species composition, abundance and diversity, Biogeosciences Discuss., 5, 2237-2278, doi:10.5194/bgd-5-2237-2008, 2008.

Reynolds, C. S.: The theory of ecological succession applied to the freshwater phytoplankton, Verh. Int. Ver. Theor. Angew. Limnol., 23, 683-691, 1988.

Reynolds, C. S.: The intermediate disturbance hypothesis and its applicability to planktonic communities: comments on the views of Padisák and Wilson, New Zealand, J. Ecol., 19, 219-225, 1995.

Reynolds, C. S.: Vegetative processes in the pelagic: A model for ecosystem theory, Ecology Institute, Oldendorf/Luhe, Germany, 1997.

Reynolds, C. S., Padisak, J., and Sommer, U.: Intermediate Disturbance in the Ecology of Phytoplankton and the Maintenance of Species Diversity: A synthesis, Hydrobiologia, 249, 183-188, 1993. 
Ruiz, J., Macías, D., and Peters, F.: Turbulence increases the average settling velocity of phytoplankton cells, Proc. Natl. Acad. Sci. U.S.A, 101, 17720-17724, 2004.

Shea, K., Roxburgh, S. H., and Rauschert, E. S. J.: Moving from pattern to process: coexistence mechanisms under intermediate disturbances regimes, Ecol. Lett., 7, 491-508, 2004.

Sherr, E. B. and Sherr, B. F.: Heterotrophic dinoflagellates: a significant component of microzooplankton biomass and majors grazers of diatoms in the sea, Mar. Ecol. Prog. Ser., 352, 187-197, 2007.

Siegel, S. and Castellan, N. J.: Non-parametric Statistics for the Behavioural Sciences, McGraw Hill Company, New York, 1988.

Sommer, U.: An experimental test of the Intermediate Disturbance Hypothesis using cultures of marine phytoplankton, Limnol. Oceanogr., 40, 1271-1277, 1995.

Sournia, A.: Le genre Ceratium (Péridinien planctonique) dans le canal du Mozambique. Contribution à une révision mondiale, Vie Milieu A: Biologie Marine 18, 375-500, 1967 (Year of publication: 1968).

Sournia, A.: Atlas du phytoplancton marin. Introduction, Cyanophycées, Dictyochophycées, Dinophycées et Raphidophycées, Vol I., Edition CNRS, Paris, 1-219, 1986.

Steidinger, K. A. and Tangen, K.: Dinoflagellates, in: Identifying Marine Phytoplankton, edited by: Tomas, C. R., Academic Press, San Diego, USA, 387-584, 1997.

Stoecker, D. K.: Mixotrophy among Dinoflagellates, J. Eukaryot. Microbiol., 46, 397-401, 1999.

Stoyanova, A. P.: New representatives of noctilucales in Bulgarian Black Sea coastal water, Compt. rend. Acad. Bulgare Sci., 52, 119-122, 1999.
Susuki, K., Nakamura, Y., and Hiromi, J.: Feeding by small calanoid copepod Paracalanus sp. on heterotrophic dinoflagellates and ciliates, Aquat. Microb. Ecol., 17, 99-103, 1999.

Throndsen, J.: Preservation and storage, edited by: Sournia, A., Phytoplankton manual, Unesco, Paris, 69-74, 1978.

Thyssen, M., Mathieu, D., Garcia, N., and Denis, M.: Short-term variation of phytoplankton assemblages in Mediterranean coastal waters recorded with an automated submerged flow cytometer, J. Plankton Res., 30, 1027-1040, 2008.

Trégouboff, G. and Rose, M.: Manuel de planctonologie méditerranéenne, Tome I, Centre National de la Recherche Scientifique, Paris, 587 pp, 1957a.

Trégouboff, G. and Rose, M.: Manuel de planctonologie méditerranéenne, Tome II, Centre National de la Recherche Scientifique, Paris, 203 pp, 1957b.

Tunin-Ley, A., Labat, J. P., Gasparini, S., Mousseau, L., and Lemée, R.: Annual cycle and diversity of species and infraspecific taxa of Ceratium Schrank (Dinophyceae) in the Ligurian Sea, NW Mediterranean, J. Phycol., 43, 1149-1163, 2007.

Venrick, E. L., McGowan, J. A., Cayan, D. R., and Hayward, T. L.: Climate and Chlorophyll $a$ : Long-Term Trends in the Central North Pacific Ocean, Science, 238, 70-72, 1987.

Vidussi, F., Marty, J. C., and Chiaverini, J.: Phytoplankton pigment variations during the transition from spring bloom to oligotrophy in the northwestern Mediterranean sea, Deep-Sea Res. Pt. 1, 47(3), 423-448, 2000.

Weithoff, G., Walz, N., and Gaedke, U.: The intermediate disturbance hypothesis-species diversity or functional diversity? J. Plankton Res., 23, 1147-1155, 2001. 